\title{
28 Research Square \\ HOXB4 promotes the malignant progression of ovarian cancer via DHDDS
}

\section{$\mathrm{Na} \mathrm{Li}$}

The first affiliated Hospital of Zunyi Medical University

Jiao Xiong

The First Affiliated Hospital of Zunyi medical University

Zhengyu Li ( $\sim 845043200 @ q q . c o m$ )

West China Second University hospital,Sichuan University https://orcid.org/0000-0003-4991-8237

\section{Research article}

Keywords: ovarian cancer, Homeobox B4, malignant progression, DHDDS

Posted Date: December 16th, 2019

DOI: https://doi.org/10.21203/rs.2.18907/v1

License: (1) This work is licensed under a Creative Commons Attribution 4.0 International License.

Read Full License

Version of Record: A version of this preprint was published at BMC Cancer on March 16th, 2020. See the published version at https://doi.org/10.1186/s12885-020-06725-4. 


\section{Abstract}

Background: Homeobox B4 (HOXB4) is associated with the poor prognosis of various cancer types. However, how HOXB4 promotes ovarian cancer (OV) progression remains to be determined. Methods $\triangle T$ The Cancer Genome Atlas (TCGA) database indicated that high level of HOXB4 in OV was correlated with poor prognosis. The biological functions of HOXB4 were confirmed through a colony formation, migration, and invasion assay. The effect of HOXB4 on the expression of EMT and cancer stem cell markers was detected. The transcriptional target of HOXB4 was DHDDS, which was detected by a ChIP assay. A xenograft tumor model was performed in nude mice to detect the role of HOXB4 in tumor proliferation and metastasis. Results $\triangle$ Results showed that the expression of HOXB4 was higher in OV tissues than in normal tissues and correlated with the poor prognosis of OV. HOXB4 knockdown suppressed the proliferation and invasion ability of OV cells in vitro. Conversely, these effects were enhanced by the up-regulation of HOXB4 in OV cells. The binding of two DNA motifs through HOXB4 regulated DHDDS expression and contributed to the malignant progression of OV. The role of HOXB4 in promoting tumor proliferation and metastasis was verified in mice. Further investigation revealed that HOXB4 triggered Snail and Zeb1 expression. Conclusion: Overall, HOXB4 overexpression was remarkably correlated with the poor prognosis of OV. HOXB4 up-regulated DHDDS, which co-contributed to the enhancer proliferation and invasion of OV cells, thus accelerating the malignant progression of OV.

\section{Background}

Ovarian cancer (OV) is predicted as the second-deadliest type of cancer among women. OV has poor prognosis in gynecologic cancer[1-4]. Though many target drugs have been applied to OV therapy, the death rate of OV is still increasing every year[5-8]. OV shows resistance to chemotherapy, radiotherapy, and molecularly targeted therapy[9]. Complex epigenetic changes pose a major challenge to OV therapy. Epithelial-mesenchymal transition (EMT) is the main malignant progression mechanism driving tumor cell metastasis and invasion[10-13]. Multiple signaling pathways and transcriptional factors, such as Snail, Zeb1, Twist, and Slug, are involved in the regulation of EMT[12-17]. New transcriptional factors, which drive EMT, should be discovered to indicate OV malignant progression.

Homeobox (HOXB) family are crucial for cell morphogenesis and differentiation[18-20]. The HOXB family involved in tumor EMT has yet to be fully investigated, and EMT HOXBs with OV have been rarely reported[18-22]. Homeobox B4 (HOXB4), an important transcription factor and involved in the progression of lung, breast, prostate, and bladder cancer[23-27]. HOXB4 enhances proliferation and stat3 pathway[28-32]. HOXB4 weakens the cytotoxic effect of paclitaxel and cisplatin by downregulating ABC transporters in OV[33]. Although HOXB4 overexpression is significantly correlated with cancer progression and poor prognosis, the precise mechanism of HOXB4 in OV remains unclear.

In this study, we revealed the potential mechanism of HOXB4 in OV malignant progression. First, we found the overexpression of HOXB4 in OV tissues is closely correlated with short survival rate in patients with OV. We also investigated that HOXB4 could promote cell proliferation, invasion, and migration of OV 
in vitro and in vivo. Overall, this study explains the mechanism of how HOXB4 regulates the malignant progression of OV.

\section{Methods}

\section{Clinical samples and TCGA database analysis}

TCGA database of OV $\mathrm{w}$ ere used to analyze gene expression and survival rate. Twenty fresh samples with adjacent normal tissues were obtained from surgical cases. The fresh tissues were used to detect the expression of HOXB4 in OV. All the patients were informed.

\section{Cell culture}

Human OV cells of SKOV3 and OVCAR3 were obtained from Shanghai Institute of Cell Biology (Cat.TCHu185\& TCHu228, Shanghai, China). Cells were maintained in DMEM (Gibco, USA) with 10\% FBS (Gibco, USA) at $37^{\circ} \mathrm{C}$ and $5 \% \mathrm{CO}_{2}$.

\section{RNA interference}

shRNAs targeting HOXB4 were purchased from Origene Biotechnology Company (Beijing, China). The Interference efficiency of shRNA was detected by Western blot after transfected for $48 \mathrm{~h}$.

\section{Colony formation assay}

Cells were seeded in a six-well plate at a final concentration of 100 cells/well. After cultured for 15 days, then the cells were fixed and stained with $0.5 \%$ crystal violet (Sigma, USA). Photographed and record the number of clones greater than 50 cells

\section{Invasion assay}

$8 \mu \mathrm{m}$ pore size transwell inserts (Millipore, USA) were used to detect cell invasion ability. Cells were added to the upper insert chamber and supplement with serum-free DMEM, and the lower culture chamber was filled with DMEM containing 20\% FBS. 36 h later, After the cells in the upper chamber are removed, the remaining invading cells are fixed and stained with crystal violet. The number of cells was counted under a light microscope (Nikon, Japan).

\section{Migration assay}

OVCAR3 and SKOV3 cells were seeded in 24-well plate and cultured for $24 \mathrm{~h}$. The linear wound was created and washed with PBS for 3 times. Then complete medium was added and culture for $36 \mathrm{~h}$. Finally, take pictures at 0,18 , and 36 hours and record the scratched area.

\section{Western blot analysis}


Total protein harvest from cells and tumor samples were separated through sodium dodecyl sulfatepolyacrylamide gel electrophoresis (SDS-PAGE) and transferred to PVDF membranes. Then membranes were blocked with $5 \%$ skimmed milk and incubated with the following specific primary antibodies at $4{ }^{\circ} \mathrm{C}$ overnight: anti-HOXB4 antibody, anti-E-cadherin, Vimentin, Snal1, and Zeb1 antibody. GAPDH was used as loading control. After washed with PBST, the membranes were incubated with HRP labeled secondary antibodies (Sigma,USA). Protein intensity was detected by Image Lab (Bio-Rad, USA).

\section{qRT-PCR}

Total RNA was extracted using TRIzol (Takara, Japan) in accordance with the manufacturer's instruction. Then, 5ug RNA was used to synthesize cDNA. Using these cDNA as template, the mRNA expression of the target gene was analyzed after 30 cycles of amplification. GAPDH was used as loading control.

\section{Luciferase reporter assay}

The DHDDS motifs were amplified from human genomic DNA and cloned into a pGL4.3 luciferase reporter vector (Promega). Transactivation assays were performed using the Dual-Luciferase Reporter Assay System (Promega). Luciferase activities were measured using a Synergy 2 microplate reader system (Gene).

\section{Zymography assays}

All media were collected and subjected to SDS-PAGE with $0.01 \% \mathrm{wt} / \mathrm{vol}$ gelatin. After electrophoresis, gels were stained with Coomassie R250 and destained until the wash became clear with apparent cleared zones associated with the MMP activity.

\section{Xenograft model}

In order to verify whether the effect of HOXB4 in animals is consistent with the results of in vitro experiments. A total of 18 6-week-old balb/c nude mice were purchased from Vital River (Beijing, China) and randomly divided into 3 groups. OVCAR3/nc, OVCAR3/HOXB4, and OVCAR3/DHDDS $\left(1 \times 10^{6}\right)$ cells were injected subcutaneously or in the tail vein. All animals were euthanized by intravenous injection of barbiturate at a final concentration of $100 \mathrm{mg} / \mathrm{kg}$, then the tumors were removed and fixed in paraffin for further analysis. The tumor volume was calculated as follows: tumor volume $=$ length $\times$ width $^{2} / 2$. All procedures performed in studies involving animals were in accordance with the ethical standards of the Institutional Animal Care and Use Committee (IACUC) at West China Second University Hospital.

\section{Histology and immunohistochemistry (IHC)}

Tumor tissue from nude mice were embedded and cut into $4 \mu \mathrm{m}$-thick sections. After microwave oven $3 \%$ $\mathrm{H}_{2} \mathrm{O}_{2}$ treatment, primary antibodies were added, HOXB4 antibody (1:500; Abcam, UK), MMP2 antibody (1:500; Abcam, UK), MMP9 antibody (1:300; Abcam, UK), E-cadherin antibody (1:500; Abcam, UK), 
vimentin, (1:500; Abcam, UK) at $4{ }^{\circ} \mathrm{C}$ overnight. The immunohistochemical staining results were collected and scored by professionals.

\section{Statistical analysis}

Statistical analyses were performed using SPSS 21.0 (SPSS Inc., USA). Statistically significant differences were analyzed using Student $t$ test, one-way ANOVA. Differences were considered significant at $\mathrm{P}<0.05$ and labeled with *

\section{Results}

\section{High level of HOXB4 is correlated with poor prognosis in OV}

The expression of HOXB4 in 373 cases of OV specimens from TCGA and 6 cases of fresh OV tissue with normal tissues were detected to examine the correlation between HOXB4 and OV prognosis. The expression level of HOXB4 was higher in OV tissues than in normal tissues. IHC results revealed the high expression level of HOXB4 in OV tumor tissues (Fig. 1a). Western blot results revealed that the protein level of HOXB4 was up-regulated in randomly selected paired tumor specimens (Fig. 1b). For TCGA database, the TPM of RNA-seq revealed the variable of HOXB4 in OV tissues (Fig. 1c). TCGA database suggested that a high expression level of HOXB4 in OV was associated with short overall survival time (Fig. 1d) and PFS (Fig. 1e). We also found that the high expression of HOXB4 in OV tissue was positively associated with clinical stage (Fig. 1f), pathologic grade (Fig. 1g), and TMB of OV (Fig. 1h). Our data verified that the aberrant overexpression of HOXB4 in OV tissues was correlated with the poor prognosis of OV.

\section{HOXB4 drives the malignant progression in TCGA analysis}

Based on the TCGA database, extract TPM from each RNA-seq, to analysis the HOXB4 high-expression group compared with the low-expression group. The GO enrichment of differentially expressed RNA revealed that embryonic development-related pathways were effectively enriched (Fig. 2a \& 2b). GSEA analysis showed that embryonic development-associated pathway was also enriched between HOXB4 high- and low-expression groups, also detect epithelial cell differentiation pathway, signal transduction of gene expression pathway and others (Fig. 2C). These data indicated that HOXB4 participated in cell differentiation and stemness and might play a malignant driving role in OV progression.

\section{HOXB4 increases OV cell malignant progression in vitro}

To further screen the expression level of HOXB4, the protein level of HOXB4 expression in various OV cell lines was detected by Western blot analysis. The HOXB4 expression level of OVCAR3 was lower but higher in SKOV3 cells (Fig. 3a). OVCAR3 cells were transfected with OVDNA3.1-HOXB4. For SKOV3 cells, shRNA was used to decrease HOXB4 expression as detected by Western blot analysis (Fig. 3b). When HOXB1 expression was up-regulated, the morphological characteristics of OVCAR3 cells changed from tightly packed colonies to a scattered growth structure (Fig. 3c). After the clone formation assay was 
conducted, quantitative analysis suggested a significant difference between high and low HOXB4 cells (Fig. 3d). In the wound healing assay, quantitative analysis suggested a significant difference in the speed of wound healing between the transfection groups and the control group (Fig. 3e). In the invasion assay (Fig. 3f), the Matrigel invasion assay revealed a sixfold increase in cell invasion in the HOXB4transfected OVCAR3 cell line compared with that in the negative vector control. MMPs act as effector molecules, which are critical in cell plasticity and EMT. The zymographic assays showed that the activities of MMP2 and MMP9 were significantly higher in the HOXB4-up-regulated group in OVCAR3 compared with those in the control group. The activity of MMP9 with HOXB4 in OVCAR3 cells was five times higher than that in the control group (Fig. 3g). For EMT markers, a high HOXB4 level was accompanied by up-regulated vimentin, Snail, and Zeb1 and downregulated E-cadherin in OVCAR3 and SKOV3 cells (Fig. 3h).

\section{HOXB4 promotes DHDDS by binding to the conserved motifs in the promoter region}

On the basis of Chip-seq in the Cistrome Data (http://dc2.cistrome.org/\#/), we referred to Yan J.'s data published in Cell, 2013, which Chip by HOXB4 in Hela cells. The top 10 target genes are shown in Fig. 4A. Furthermore, we screened the binding motif by HOXB4 for the top one, DHDDS, by Wash U analysis (http://epigenomegateway.wustl.edu/legacy/?

genome=hg38\&datahub=http://dc2.cistrome.org/api/datahub/42672\&coordinate=chr1:532324-732413). We defined motifs based on the Chip-seq peaks in the DHDDS promoter region by using the ChIPseeker online software through motif analysis. Two individual motifs and their locations within the promoter of DHDDS were found. Motif1 and Motif2 possessed the relatively conserved sequences of Chr1 629791630040 and Chr1 633904-634132 (Fig 4B). Then, we used the up-regulated expression of HOXB4 in OVCAR3 cells and knocked down HOXB4 in SKOV3 cells to detect DHDDS expression levels. The results showed that DHDDS expression was up-regulated follow HOXB4 high levels (Fig. 4C). The truncated luciferase report plasmids were used for motif identification. The DHDDS motifs were truncated into two regions, which one was covered its peak sequence. The luciferase assay data showed that all HOXB4 transcriptionally activated motifs 1 and 2 and enhancer by $1+2$ sequences, indicating that motif 1 might synergize motif2 in transcriptional activation by HOXB4 regulation (Fig. 4D). To further confirm the transcriptional activation of DHDDS by HOXB4, we analyzed the correlation between HOXB4 and DHDDS expression in the HCC samples of the TCGA database. In TCGA, OV data were downloaded and analyzed using R pack, and the results indicated that HOXB4 was positively correlated with DHDDS, R=0.12, $p=0.051$ (Fig. 4E). Furthermore, we examined the tumor sizes and node numbers, which was significantly related to the DHDDS level (Fig. 4FG). However, clinical stage and grade had no significant relationship with DHDDS, suggesting that DHDDS might participate in the complex process of PAAD progression via the downstream target gene.

\section{HOXB4 relies on DHDDS to promote OV cell progression}

RNA-seq GO and GSEA analysis indicated that malignant progression, such as proliferation, migration, invasion, and EMT ability, may be enhanced by HOXB4 in OV. We then identified the biological function of 
HOXB4 in OV cells. We used OVCAR3 and SKOV3 cells to evaluate the biological function of HOXB4 in OV process. To assess the effect of HOXB4/DHDDS on the growth of OV, we overexpressed HOXB4 and DHDDS in OVCAR3 cells and knocked them down via shRNA in SKOV3 cells. For the recovery experiment, we used HOXB4 overexpression with knocked down DHDDS by antisense oligonucleotide (KO) in OVCAR3 or knocked down HOXB4 by shRNA with DHDDS-overexpressing mimic in SKOV3 cells. The results showed that HOXB4 overexpression or DHDDS mimic promoted proliferation by the colony formation assay in OVCAR3 (Fig. 5A). Conversely, the proliferation of SKOV3 was inhibited by HOXB4 downregulation by shRNA and DHDDS KO (Fig.5B). When block DHDDS follow HOXB4, or enhance DHDDS to reverse effect follow HOXB4 down-regulated, shown same neutralizing effect in the two cells. The effect of HOXB4 on the migration and invasion abilities of OV cells was examined. Wound healing assays showed that HOXB4 and DHDDS overexpression enhanced cell migration as presented by a narrowing gap (Fig. 5B). Conversely, knocked down HOXB4 and DHDDS KO weakened the cell migration. For the invasion assay, transwell assays showed effects similar to those of the wound healing assay. HOXB4 and DHDDS promoted invasion in OV cells, knockdown HOXB4 and inhibit DHDDS weakness the effect (Fig. 5C). These results indicated that HOXB4 presented its effect on malignant progression dependent on DHDDS overexpression, which supported the transcriptional activation relationship between HOXB4 and DHDDS.

\section{HOXB4 facilitates the progression of OV in the mouse model}

To further validate the role of HOXB4 in OV growth and metastasis, we established an OVCAR3 xenograft model by using Balb/c-nu/nu mice. An OVCAR3 stable transfection clone was obtained by G418 enrichment and then injected subcutaneously or in the tail vein of the mice. When HOXB4 and DHDDS were individually overexpressed, tumor growth was significantly enhanced. Conversely, when blocked HOXB4 downstream DHDDS, the growth of the tumor was inhibited (Fig. 6AB). The quantification of the number of lung metastatic nodes showed that HOXB4 and DHDDS conferred more colonize in the lung (Fig. 6C). Survival analysis revealed that HOXB4 overexpression in OV contributed to a short survival time, and the up-regulation of DHDDS also worsened the outcome (Fig. 6D). The IHC staining of tumor tissues demonstrated invasion and metastasis, and EMT markers were up-regulated follow overexpression HOXB4 and DHDDS. Thus, HOXB4 accelerated OV progression depending on its target DHDDS.

\section{Discussion}

HOXB4 plays important role in proliferation, metastasis, and angiogenesis in cancer [23-25, 28, 33-40]. Our results indicated that HOXB4 was overexpressed in OV tissues and correlated with the poor prognosis of patients with OV. The overexpression of HOXB4 promoted cell proliferation and invasion of OV both in vitro and in vivo. The transcriptional target of HOXB4 was DHDDS. Our results suggested that HOXB4 promoted the invasion and EMT of OV cells to accelerate the malignant progression of OV by upregulating DHDDS. 
HOXB4 over-expression has been demonstrated in OPSCC cancer, indicating poor prognosis in patients. The up-regulation of HOXB4 in atypical myeloproliferative neoplasms was associated with the malignant progression of the cancer [23]. HOXB4 can modulate OV chemotherapy resistance[33]. However, how HOXB4 regulates the progression of OV is unclear. Our study confirmed that HOXB4 was associated with the poor prognosis of OV and revealed that HOXB4 enhanced EMT properties in OV cells.

Previous studies indicated that HOXB4 plays an crucial role in cancer progression [24, 32, 41-44]. EMT is important during tumor metastasis that epithelial cells to lose adhesion and acquire invasive ablibity [4548]. HOXB4 induced EMT and contributed to migration breast cancer cell metastasis. Knockdown of HOXB4 inhibited the EMT related invasion in lung cancer. Our results demonstrated that HOXB4 had a substantial effect on the EMT phenotypes, thus enhancing OV cell migration and invasion in OV.

Although HOXB4 in the progression of OV has been studied, the specific mechanism of HOXB4 in regulating $\mathrm{OV}$ remains unclear. Further investigation showed that the transcriptional activity of DHDDS expression was up-regulated by HOXB4. This indicated that the effect of HOXB4 in OV may mediated by noncoding RNAs. Our results showed that the HOXB4/DHDDS axis was modulated by direct transcriptional regulation to promote invasion in OV cells. In addition, our results revealed that HOXB4 promoted OV cell metastasis and contributed to Snail and Zeb1 expression. This finding suggested that HOXB4 promoted the metastasis of OV cells by activating EMT-associated pathways.

\section{Conclusions}

In brief, HOXB4 promoted the malignant progression of OV by regulating DHDDS. Our study verified that HOXB4 enhanced OV cell metastasis by promoting EMT and facilitated OV growth by promoting stemness. These results implied that HOXB4 could regulate several signaling pathways, but DHDDS was the primary target activated by HOXB4 to modulate the malignant progression of OV.

\section{List Of Abbreviations}

Homeobox B4 (HOXB4); ovarian cancer (OV); The Cancer Genome Atlas (TCGA); Epithelial mesenchymal transition (EMT)

\section{Declarations}

Ethics approval and consent to participate: All animal experiments in this study were confirmed that ethical and legal approval was obtained prior to the commencement of the study. All procedures performed in studies involving animals were in accordance with the ethical standards of the Institutional Animal Care and Use Committee (IACUC) at West China Second University Hospital.

Consent for publication: Not applicable

Availability of data and materials: Not applicable 
Competing interests: The authors declare that they have no competing interests

Funding: Guizhou Science and Technology Cooperation Program 20157450, funding body LN is responsible for article writing and cell experiments.

Authors' contributions: LZY is responsible for experimental design and article writing, $L N$ is responsible for article writing and cell experiments, and $\mathrm{XJ}$ is responsible for data analysis and animal experiments. All authors read and approved the final manuscript.

Acknowledgements: Not applicable

Author details: 1.Department of Obstetrics and Gynecology, Key Laboratory of Birth Defects and Related Diseases of Women and Children, Ministry of Education, West China Second University Hospital, Sichuan University, Chengdu 610041, People's Republic of China. 2. Department of Obstetrics and Gynecology, The first affiliated Hospital of Zunyi Medical University, Zunyi 563000, People's Republic of China

\section{References}

1. Yang J, Xing H, Lu D, Wang J, Li B, Tang J, Gu F, Hong L: Role of Jagged1/STAT3 signalling in platinum-resistant ovarian cancer. J Cell Mol Med 2019.

2. Suidan RS, He W, Sun CC, Zhao H, Rauh-Hain JA, Fleming ND, Lu KH, Giordano SH, Meyer LA: Total and out-of-pocket costs of different primary management strategies in ovarian cancer. Am J Obstet Gynecol 2019.

3. Morgan RD, Stamatopoulou S, Mescallado N, Saunders G, Welch R, Mitchell C, Hasan J, Clamp AR, Jayson GC: Screening tool for malignant bowel obstruction in relapsed, metastatic ovarian cancer. ESMO Open 2019, 4(2):e000463.

4. Luh L, Nyoman BMI, Aag PW, Ketut A, Ayu B: Ovarian Cancer Immature Teratoma Type in Pregnancy: Management and Feto-Maternal Outcomes. Open Access Maced J Med Sci 2019, 7(6):1016-1020.

5. Liu Y, Chanana P, Davila JI, Hou X, Zanfagnin V, McGehee CD, Goode EL, Polley EC, Haluska P, Weroha SJ et al: Gene expression differences between matched pairs of ovarian cancer patient tumors and patient-derived xenografts. Sci Rep 2019, 9(1):6314.

6. Gentiluomo M, Villa C, Sommavilla E, Pesenti S, Parimbelli L, Bambina S, Valerii C, Sironi S: Inflammatory breast metastasis from primary advanced ovarian cancer. Breast $J$ 2019, 25(3):507509.

7. Garcia C, Harrison K, Ring KL, Sullivan MW, Rauh LA, Modesitt SC: Genetic counseling referral for ovarian cancer patients: a call to action. Fam Cancer 2019.

8. Daniele G, Arenare L, Scambia G, Pisano C, Sorio R, Breda E, De Placido S, Savarese A, Ferrandina G, Raspagliesi $\mathrm{F}$ et al: Prognostic role of chemotherapy-induced neutropenia in first-line treatment of advanced ovarian cancer. A pooled analysis of MITO2 and MITO7 trials. Gynecol Oncol 2019. 
9. Cabasag CJ, Arnold M, Butler J, Inoue M, Trabert B, Webb PM, Bray F, Soerjomataram I: The influence of birth cohort and calendar period on global trends in ovarian cancer incidence. Int J Cancer 2019.

10. Ye X, Brabletz T, Kang Y, Longmore GD, Nieto MA, Stanger BZ, Yang J, Weinberg RA: Upholding a role for EMT in breast cancer metastasis. Nature 2017, 547(7661):E1-E3.

11. Pearlman RL, Montes de Oca MK, Pal HC, Afaq F: Potential therapeutic targets of epithelialmesenchymal transition in melanoma. Cancer Lett 2017, 391:125-140.

12. Nieto MA, Huang RY, Jackson RA, Thiery JP: Emt: 2016. Cell 2016, 166(1):21-45.

13. Chaffer CL, San Juan BP, Lim E, Weinberg RA: EMT, cell plasticity and metastasis. Cancer Metastasis Rev 2016, 35(4):645-654.

14. Antony J, Huang RY: AXL-Driven EMT State as a Targetable Conduit in Cancer. Cancer Res 2017, 77(14):3725-3732.

15. Han J, Meng J, Wang X, Yin S, Zhang Q, Liu H, Qin R, Li Z, Zhong W, Zhang C et al: YY1 complex promotes Quaking expression via super-enhancer binding during EMT of hepatocellular carcinoma. Cancer Res 2019.

16. Meng J, Chen S, Han JX, Qian B, Wang XR, Zhong WL, Qin Y, Zhang H, Gao WF, Lei YY et al: Twist1 Regulates Vimentin through Cul2 Circular RNA to Promote EMT in Hepatocellular Carcinoma. Cancer Res 2018, 78(15):4150-4162.

17. Sun T, Zhao N, Zhao XL, Gu Q, Zhang SW, Che N, Wang XH, Du J, Liu YX, Sun BC: Expression and functional significance of Twist1 in hepatocellular carcinoma: its role in vasculogenic mimicry. Hepatology 2010, 51(2):545-556.

18. Kam MK, Lui VC: Roles of Hoxb5 in the development of vagal and trunk neural crest cells. Dev Growth Differ 2015, 57(2):158-168.

19. Xavier FC, Destro MF, Duarte CM, Nunes FD: Epigenetic repression of HOXB cluster in oral cancer cell lines. Arch Oral Biol 2014, 59(8):783-789.

20. Vieux-Rochas M, Mascrez B, Krumlauf R, Duboule D: Combined function of HoxA and HoxB clusters in neural crest cells. Dev Bio/ 2013, 382(1):293-301.

21. Tucci R, Campos MS, Matizonkas-Antonio LF, Durazzo M, Pinto Junior Ddos S, Nunes FD: HOXB5 expression in oral squamous cell carcinoma. J App/ Oral Sci 2011, 19(2):125-129.

22. Liao WT, Jiang D, Yuan J, Cui YM, Shi XW, Chen CM, Bian XW, Deng YJ, Ding YQ: HOXB7 as a prognostic factor and mediator of colorectal cancer progression. Clin Cancer Res 2011, 17(11):35693578.

23. Dumas PY, Mansier O, Prouzet-Mauleon V, Koya J, Villacreces A, Brunet de la Grange P, Luque Paz D, Bidet $\mathrm{A}$, Pasquet JM, Praloran $\mathrm{V}$ et al: MiR-10a and HOXB4 are overexpressed in atypical myeloproliferative neoplasms. BMC Cancer 2018, 18(1):1098.

24. Bonfim-Silva R, Ferreira Melo FU, Thome CH, Abraham KJ, De Souza FAL, Ramalho FS, Machado HR, De Oliveira RS, Cardoso AA, Covas DT et al: Functional analysis of HOXA10 and HOXB4 in human medulloblastoma cell lines. Int J Oncol 2017, 51(6):1929-1940. 
25. Wang H, Jia XH, Chen JR, Yi YJ, Wang JY, Li YJ, Xie SY: HOXB4 knockdown reverses multidrug resistance of human myelogenous leukemia K562/ADM cells by downregulating P-gp, MRP1 and BCRP expression via PI3K/Akt signaling pathway. Int J Onco/ 2016, 49(6):2529-2537.

26. Dos Santos Schiavinato JL, Oliveira LH, Araujo AG, Orellana MD, de Palma PV, Covas DT, Zago MA, Panepucci RA: TNF-alpha and Notch signaling regulates the expression of HOXB4 and GATA3 during early T lymphopoiesis. In Vitro Cell Dev Biol Anim 2016, 52(9):920-934.

27. Fournier M, Lebert-Ghali CE, Bijl JJ: HOXA4 provides stronger engraftment potential to short-term repopulating cells than HOXB4. Stem Cells Dev 2015, 24(20):2413-2422.

28. Teichweyde N, Kasperidus L, Carotta S, Kouskoff V, Lacaud G, Horn PA, Heinrichs S, Klump H: HOXB4 Promotes Hemogenic Endothelium Formation without Perturbing Endothelial Cell Development. Stem Cell Reports 2018, 10(3):875-889.

29. Huang X, Lee MR, Cooper S, Hangoc G, Hong KS, Chung HM, Broxmeyer HE: Activation of OCT4 enhances ex vivo expansion of human cord blood hematopoietic stem and progenitor cells by regulating HOXB4 expression. Leukemia 2016, 30(1):144-153.

30. Hong SH, Yang SJ, Kim TM, Shim JS, Lee HS, Lee GY, Park BB, Nam SW, Ryoo ZY, Oh IH: Molecular integration of HoxB4 and STAT3 for self-renewal of hematopoietic stem cells: a model of molecular convergence for stemness. Stem Cells 2014, 32(5):1313-1322.

31. Fujiwara T, Yokoyama H, Okitsu Y, Kamata M, Fukuhara N, Onishi Y, Fujimaki S, Takahashi S, Ishizawa K, Bresnick EH et al: Gene expression profiling identifies HOXB4 as a direct downstream target of GATA-2 in human CD34+ hematopoietic cells. PLoS One 2012, 7(9):e40959.

32. Fan R, Bonde S, Gao P, Sotomayor B, Chen C, Mouw T, Zavazava N, Tan K: Dynamic HoxB4-regulatory network during embryonic stem cell differentiation to hematopoietic cells. Blood 2012, 119(19):e139147.

33. Li Y, Sun J, Gao S, Hu H, Xie P: HOXB4 knockdown enhances the cytotoxic effect of paclitaxel and cisplatin by downregulating ABC transporters in ovarian cancer cells. Gene 2018, 663:9-16.

34. Gaunt SJ: Mouse embryo Hox gene enhancers assayed in cell culture: Hoxb4, b8 and a7 are activated by Cdx1 protein. Int J Dev Biol 2018, 62(11-12):717-722.

35. Li L, Zhao CT, Cui BL, Wu SL, Liu XD, Su Z, Yang J, Wang W, Cui ZG, Zhao HG: [Expression of HOXB4, PRDM16 and HOXA9 in Patients with Acute Myeloid Leukemia and Its Clinical Significance]. Zhongguo Shi Yan Xue Ye Xue Za Zhi 2016, 24(2):326-331.

36. Jorgensen S, Coskun M, Homburg KM, Pedersen OB, Troelsen JT: HOXB4 Gene Expression Is Regulated by CDX2 in Intestinal Epithelial Cells. PLoS One 2016, 11(10):e0164555.

37. Nanbakhsh A, Pochon C, Amsellem S, Pittari G, Tejchman A, Bourhis JH, Chouaib S: Enhanced cytotoxic activity of ex vivo-differentiated human natural killer cells in the presence of HOXB4. $J$ Immunother 2014, 37(5):278-282.

38. Qiao Y, Zhao CT, Liu ZZ, Feng XQ, Wang L, Liu SH: Construction of lentivirus vector containing human homeobox gene HOXB4 and its expression in human umbilical cord mesenchymal stem cells. Zhongguo Shi Yan Xue Ye Xue Za Zhi 2012, 20(3):703-709. 
39. Park SW, Won KJ, Lee YS, Kim HS, Kim YK, Lee HW, Kim B, Lee BH, Kim JH, Kim DK: Increased HoxB4 Inhibits Apoptotic Cell Death in Pro-B Cells. Korean J Physiol Pharmacol 2012, 16(4):265-271.

40. Forrester LM, Jackson M: Mechanism of action of HOXB4 on the hematopoietic differentiation of embryonic stem cells. Stem Cells 2012, 30(3):379-385.

41. Deng C, Li Y, Liang S, Cui K, Salz T, Yang H, Tang Z, Gallagher PG, Qiu Y, Roeder R et al: USF1 and hSET1A mediated epigenetic modifications regulate lineage differentiation and HoxB4 transcription. PLoS Genet 2013, 9(6):e1003524.

42. Wen-jun L, qu-lian G, Hong-ying C, Yan Z, Mei-Xian H: Studies on HOXB4 expression during differentiation of human cytomegalovirus-infected hematopoietic stem cells into lymphocyte and erythrocyte progenitor cells. Cell Biochem Biophys 2012, 63(2):133-141.

43. Tashiro K, Kawabata K, Omori M, Yamaguchi T, Sakurai F, Katayama K, Hayakawa T, Mizuguchi H: Promotion of hematopoietic differentiation from mouse induced pluripotent stem cells by transient HoxB4 transduction. Stem Cell Res 2012, 8(2):300-311.

44. Jackson M, Axton RA, Taylor AH, Wilson JA, Gordon-Keylock SA, Kokkaliaris KD, Brickman JM, Schulz H, Hummel O, Hubner N et al: HOXB4 can enhance the differentiation of embryonic stem cells by modulating the hematopoietic niche. Stem Cells 2012, 30(2):150-160.

45. Liang H, Zhao X, Wang C, Sun J, Chen Y, Wang G, Fang L, Yang R, Yu M, Gu Y et al: Systematic analyses reveal long non-coding RNA (PTAF)-mediated promotion of EMT and invasion-metastasis in serous ovarian cancer. Mol Cancer 2018, 17(1):96.

46. Yan J, Zhang Y, Shi W, Ren C, Liu Y, Pan Y: The critical role of HMGA2 in regulation of EMT in epithelial ovarian carcinomas. Tumour Biol 2016, 37(1):823-828.

47. Gao J, Zhu Y, Nilsson M, Sundfeldt K: TGF-beta isoforms induce EMT independent migration of ovarian cancer cells. Cancer Cell Int 2014, 14(1):72.

48. Wu J, Liu Z, Shao C, Gong Y, Hernando E, Lee P, Narita M, Muller W, Liu J, Wei JJ: HMGA2 overexpression-induced ovarian surface epithelial transformation is mediated through regulation of EMT genes. Cancer Res 2011, 71(2):349-359.

\section{Figures}

\section{Figure 1}

HOXB4 up-regulation in OV tissues was correlated with poor prognosis. A. IHC staining showed the high and low HOXB4 expression levels in OV tissues. B. Representative WB revealed the expression level of HOXB4 in OV and paired adjacent noncancerous tissues. C. HOXB4 was negative in adjacent noncancerous tissue and positive in OV tissue. D. Kaplan-Meier survival analysis of OS in patients with OV. A high level of HOXB4 resulted in a short survival time in patients with OV; a low level of HOXB4 was correlated with a long survival time $(P<0.05)$. E. PFS in OV showed results similar to OS. F. HOXB4 was 
positive in high grades in OV $(\mathrm{P}<0.05)$. G. HOXB4 was positively correlated with a tumor mutational burden $(T M B ; P<0.05)$. H. HOXB4 was positively correlated with lymph node metastasis $(P<0.05)$.

\section{Figure 2}

Bioinformatics analysis of patients with HOXB4 in TCGA with high expression in patients and low expression, express the differences between the spectra. A. GO analysis showed that pathways associated with embryo morphogenesis were enriched. B. GSEA analysis revealed that multicancer invasiveness and embryonic stem cell core were enriched. C. Metascape analysis also enriched embryonic-associated pathways.

\section{Figure 3}

HOXB4 facilitated OV cell proliferation by promoting progression. A. Protein level of HOXB4 in different OV lines. B. Efficiency of HOXB4 knockdown and overexpression in SKOV3 and OVCAR3 cells, respectively. C. Clonal morphological characteristics of HOXB4 in OVCAR3 and SKOV3 cells. D. Cell colony formation assay was used to examine the effect of HOXB4 on the proliferation of OV cells. HOXB4 promoted the proliferation of OV cells $(\mathrm{P}<0.05$, respectively). E. Analysis of wound-healing assay in OVCAR3 and SKOV3 cells. HOXB4 promoted the migration of OV cells $(\mathrm{P}<0.05$, respectively). F. Analysis of invasion by Transwell assay in OVCAR3 and SKOV3 cells. HOXB4 accelerated the invasion of OV cells $(P<0.05$, respectively). $G, H$. Zymography assays and WB to detect invasion and EMT marker expression levels after HOXB4 knockdown and overexpression.

\section{Figure 4}

Motifs regulated by HOXB4 in its target DHDDS. A. Top 10 target analysis by Chip-seq in Cistrome Data (http://dc2.cistrome.org/\#/). B. Motif analysis of the target gene DHDDS promoter region by using the ChIPseeker. Two individual motifs and their locations within the promoter of DHDDS, Chr1 629791630040, and Chr1 633904-634132, respectively. C. qRT-PCR was employed to detect the level of DHDDS after HOXB4 knockdown and overexpression; HOXB4 promoted DHDDS expression ( $\mathrm{P}<0.05)$. D. Luciferase report assay showed that all HOXB4 transcriptionally activated motifs 1 and 2 and enhanced by $1+2$ sequences. E. TCGA database showed a positive correlation between HOXB4 and DHDDS expression in the OV samples. F. HOXB4 was positively correlated with DHDDS, R=0.12, $p=0.051$. G. Tumor sizes and node numbers were significantly related to the DHDDS level $(P<0.05)$.

\section{Figure 5}


Cell function analysis of the effect of HOXB4/DHDDS of OV. A. Colony formation assay detected the overexpressed HOXB4, DHDDS promoted the proliferation of OVCAR3 cells, and knockdown by shRNA suppressed the proliferation of SKOV3 cells. HOXB4 overexpression with CRIPSR DHDDS by the cas9 system (KO) in OVCAR3 or HOXB4 knockdown by shRNA with a DHDDS-overexpressing mimic in SKOV3 cells elicited neutralizing effects. B. Wound-healing assay. C. Invasion assay revealed results similar to those of A. HOXB4 transcriptional activation DHDDS promoted cell progression.

\section{Figure 6}

Effect of HOXB4 on the growth and metastasis of OV cells in vivo. A. Representative images of tumor in the OVCAR3/scramble, OVCAR3/HOXB4, OVCAR3/DHDDS, and OVCAR3/HOXB4 + DHDDS-KO groups (n $=6$ per group). B. Tumor volume follow days, HOXB4 and DHDDS overexpression promoted tumor growth. Quantification of fluorescence from metastatic tumors $(P<0.01$, respectively). C. H\&E staining of metastatic tumors in lung tissues and node counts; HOXB4 and DHDDS promoted lung metastasis. D. Kaplan-Meier survival analysis of mice in different groups. E. IHC staining of tumor samples and gray analysis of positive area to detect the progression markers for each group.

\section{Supplementary Files}

This is a list of supplementary files associated with this preprint. Click to download.

- NC3RsARRIVEGuidelines2013.pdf 